\title{
Wild-type but not mutant SOD1 transgenic astrocytes promote the efficient generation of motor neuron progenitors from mouse embryonic stem cells
}

Yiota A Christou ${ }^{1 *}$, Kyoji Ohyama ${ }^{3}$, Marysia Placzek ${ }^{3}$, Peter N Monk ${ }^{2+}$ and Pamela J Shaw ${ }^{1+}$

\begin{abstract}
Background: The efficient derivation of mature $(\mathrm{Hb} 9+)$ motor neurons from embryonic stem cells is a sought-after goal in the understanding, and potential treatment, of motor neuron diseases. Conditions that promote the robust generation of motor neuron progenitors from embryonic stem cells and that promote the survival of differentiated motor neurons ex vivo are likely, therefore, to be critical in future biological/therapeutic/screening approaches. Previous studies have shown that astrocytes have a protective effect on differentiated motor neurons (in vivo and ex vivo), but it remains unclear whether astrocytes also play a beneficial role in the support of motor neuron progenitors. Here we explore the effect of murine astrocyte-conditioned medium on monolayer cultures of mouse embryonic stem cell-derived motor neuron progenitors.

Results: Our data show that wild-type astrocyte-conditioned medium significantly increases the number of Olig2+/Hb9- progenitors, which subsequently differentiate into Hb9+/lslet1+ post-mitotic motor neurons. Intriguingly, while astrocyte-conditioned medium derived from mice transgenic for wild-type human SOD1 mimics the effect of wild-type astrocytes, conditioned medium derived from astrocytes carrying an amyotrophic lateral sclerosis-related SOD1-G93A mutation shows no such beneficial effect. The effect of astrocyte-conditioned medium, moreover, is specific to motor neurons: we find that interneurons generated from mouse embryonic stem cells are unaffected by conditioned media from any type of astrocyte.
\end{abstract}

Conclusions: Our study indicates that conditioned medium derived from wild type astrocytes enhances the efficient generation of motor neurons from mouse embryonic stem cells by enhancing motor neuron progenitors. In contrast, conditioned medium from SOD1-G93A astrocytes does not show a similar enhancing effect.

Keywords: ES cells, G93A astrocytes, Motor neuron, Progenitor, Differentiation, ALS

\section{Background}

Motor neurons (MNs) are selectively depleted, or diminished, in motor neuron diseases such as amyotrophic lateral sclerosis (ALS). Various factors contribute to the pathogenesis of ALS including mutations in the SOD1 gene, mutations in the ALS2 (Alsin) [1,2], FUS (RNA-binding protein FUS) [3], TARDBP (TAR DNA-binding protein 43) [4,5], ATXN2 (Ataxin-2) [6] and ANG (Angiogenin) [7]

\footnotetext{
*Correspondence: yiotachrist17@hotmail.com

${ }^{\dagger}$ Equal contributors

'Department of Neuroscience, Sheffield Institute for Translational Neuroscience (SITraN), University of Sheffield, Sheffield, S10 2HQ, UK Full list of author information is available at the end of the article
}

genes, and the recently-discovered intronic hexanucleotide expansions in C9ORF72 [8,9]. In mutant SOD1 disease models, non-neuronal cells, such as astrocytes have been implicated in the pathogenesis of $\mathrm{MN}$ degeneration [10-12]. The mechanism by which astrocytes facilitate MN loss has not yet been fully identified, but a number of potential mechanisms have been reported. The production of NGF (nerve growth factor) (or related species such as NGF-precursor forms (pro-NGF)) in reactive SOD1G93A (change of alanine to glycine at position 93 of the SOD1 protein) astrocytes can induce death of rat MNs expressing p75-receptor, through a mechanism that involves

\section{Biomed Central}


nitric oxide and peroxynitrite formation [13]. The increased secretion of pro-NGF and subsequent induction of toxic p75 receptor signalling within neighbouring motor neurons is believed to be a key element of the toxicity of mutant SOD1-expressing astrocytes to motor neurons [14]. Studies in mouse have similarly shown that astrocytes expressing mutant forms of SOD1-G37R (change of arginine to glycine at position 37), SOD1-G85R (change of arginine to glycine at position 85), and SOD1-G93A (change of alanine to glycine at position 93), release soluble factors that can selectively kill embryonic stem (ES) cell-derived or primary MNs in vitro, and have demonstrated that neurotoxicity by SOD1-G93A astrocytes is mediated through the recruitment of the Bax-dependent death machinery. By contrast, conditioned medium from wild-type SOD1expressing astrocytes displays a supportive/survival effect on MNs similar to that observed with non-transgenic astrocytes [15]. Co-culture of ES cell-derived MNs with SOD1-G93A astrocytes markedly decreases MN survival relative to primary wild-type $S O D 1$ astrocytes [16]. In vivo, the transplantation of glial-restricted precursor cells into the vicinity of cervical spinal cord respiratory MNs in a SOD1-G93A rat model of motor neuron disease limits progression of the disease, resulting in enhanced motor and respiratory physiological functions and enhanced survival [17]. The neuroprotective effects have been partly attributed to increased expression of the astrocytic glutamate transporter, GLT1. Taken together, the evidence from these studies suggests that astrocytes are critically involved in MN depletion in ALS, most likely acting through multiple mechanisms.

As yet, no study has examined whether astrocytes exert an effect on MN-progenitor cells. Two factors suggest this is an important question. First, the efficient derivation of mature $(\mathrm{Hb} 9+)$ MNs from embryonic stem cells is a sought-after goal in the understanding, and potential treatment, of motor neuron diseases: factors that enhance early steps in MN differentiation will therefore contribute to the derivation of MNs ex vivo. Second, the realisation that new neurons can be generated within the adult brain suggests the possibility that ALS-driven MN depletion may result from the loss of adult-born neurons. Recent experiments in songbirds show that adult neurogenesis can result in the addition of new neurons to cortical premotor circuits that govern peripheral muscle control [18], while dopamine produced by projections of the brain promotes adult MN regeneration in lesioned spinal cord in zebrafish [19], supporting this idea. Thus, we aimed to extend current knowledge by examining the potential effect of astrocytes on $\mathrm{MN}$ progenitors. Here we show that wild-type astrocyte-conditioned medium promotes the efficient generation of $\mathrm{MN}$ progenitors from mouse ES cells. By contrast, SOD1-G93A astrocytes are less supportive for the generation of these $\mathrm{MN}$ progenitors.

\section{Results}

Spatio-temporal expression profiles of transcription factors in MN development in mouse neural tube

As a basis for monitoring MN differentiation from mouse ES cells in vitro, we first examined the expression profiles of the transcription factors Olig2, Hb9/MNR2 and Islet1 in the embryonic mouse neural tube at E10.5; all have been previously demonstrated to be involved in $\mathrm{MN}$ fate determination [20-22].

As previously shown [20-22], Olig2 expression is restricted to the ventral neural tube, demarcating progenitor domain (p)V3 and (p)MN (Figure 1). By contrast, Hb9/ MNR2 expression is restricted to differentiated MNs in the mantle zone of the emerging spinal cord (Figure 1). Consistent with this, double labelling of Hb9/MNR2 with Ki67, a marker of proliferating cells, reveals no overlap, confirming that Hb9/MNR2 is a marker for post-mitotic MNs (Figure 1). Double-labelling for Olig2 and $\mathrm{Hb} 9 /$ MNR2 reveals that the majority of Olig2+ cells do not co-express Hb9/MNR2 and are largely restricted to the ventricular zone and subventricular zone (VZ/SVZ), the region of the developing neural tube that harbours proliferating progenitor cells. A small proportion of Olig2+ cells (8.47\%) were Hb9/MNR2+. These Olig2+/Hb9+ cells were found furthest from the VZ/SVZ, and represent a population of Olig2+ cells that are making their transition towards Hb9/MNR2+ differentiating MNs as they migrate away from the medial $\mathrm{VZ}$ to the peripheral mantle zone (Figure 1). Similarly, a few Olig2+/Islet1+ cells were located away from the VZ/SVZ and were detected immediately adjacent to Olig2-/Islet1+ differentiated MNs (Figure 1). Taken together, our data confirm previous reports [23,24], showing that many Olig2+ cells are proliferating MN progenitor cells. These analyses suggest, further, that the combinatorial analysis of Olig2, Hb9/MNR2 and Islet1 expression can be used as a basis for monitoring the differentiation status of MNs from mouse ES cells.

\section{Wild-type but not mutant SOD1-G93A astrocyte-conditioned medium promotes the generation of MN progenitors from mouse ES cells and subsequent $\mathrm{MN}$ differentiation}

The effect of conditioned medium (CM) from cultured wild-type non-transgenic (wt non-Tg) astrocytes on differentiated MNs was first assessed, in comparison to control medium (not conditioned by astrocytes). MN differentiation was assessed through Hb9:eGFP reporter activity and further confirmed by co-expression of either $\beta 3$ tubulin or Islet1. Hb9:eGFP+ MNs co-expressed $\beta 3-$ tubulin (Figure 2a,b; white arrows: 100\% co-expression, data not shown) and Islet1 (Figure 2e-g; white arrows: 95.03\% co-expression, data not shown). Quantitative analyses showed that culture with wt non-Tg astrocyte CM resulted in a marked increase in MNs. The percentage of eGFP+ MNs was increased by a fold-change of 2.8, 

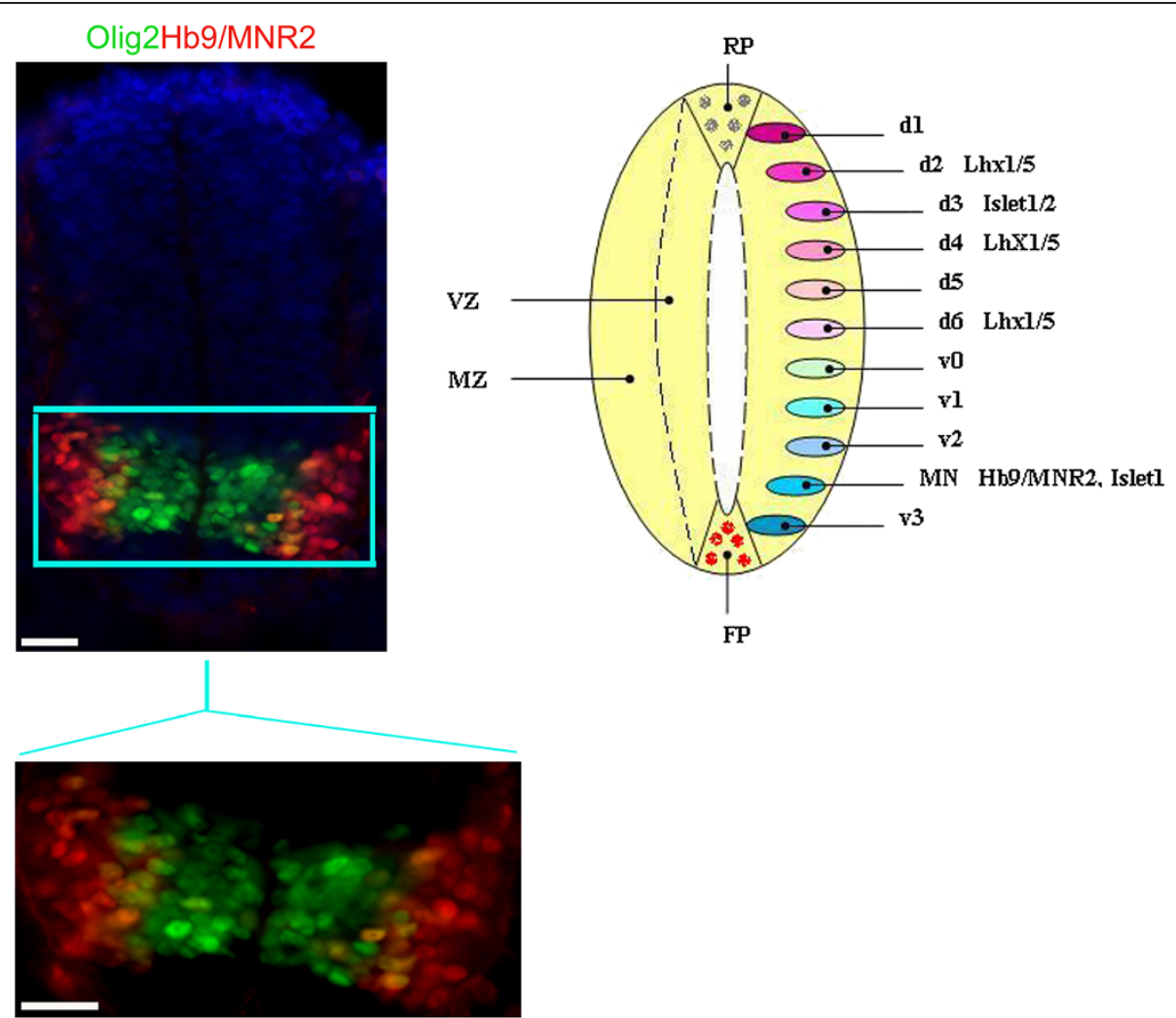

Olig2lslet1

Ki67Hb9/MNR2
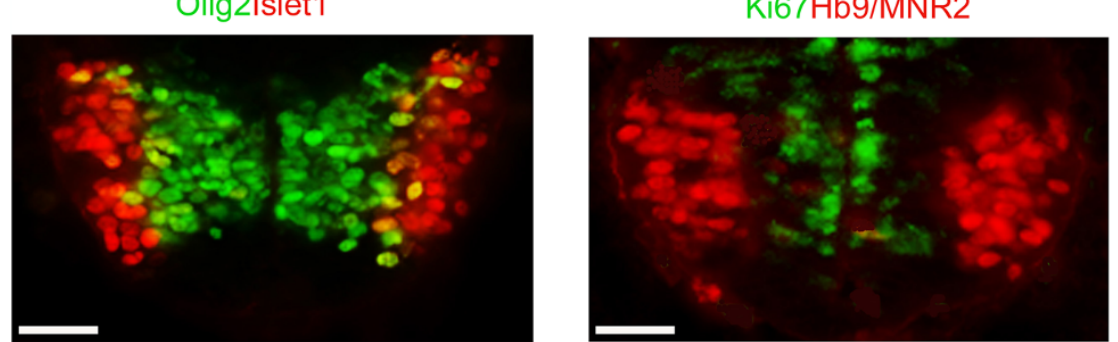

Figure 1 Motor neuron development in embryonic mouse neural tube. Fluorescence images of embryonic (E10.5) mouse neural tube, after immunolabelling for Olig2 (green), Islet1 (red), Hb9/MNR2 (red), Ki67 (green) and DAPI for nuclei (blue). Scale bars $50 \mu m$. The schematic of the neural tube shows the dorsal $(\mathrm{d} 1-\mathrm{d} 6)$ and ventral domains $(\mathrm{v} 0-\mathrm{v} 3, \mathrm{MN})$ of the neural tube and corresponding expression markers. RP: roof plate, FP: floor plate, VZ: ventricular zone, MZ: mantle zone, d: dorsal, v: ventral, MN: motor neuron. Olig2+ cells are restricted to the VZ, the region of proliferating progenitor cells in the developing neural tube. Islet1+ and Hb9/MNR2+ cells are found at the peripheral mantle zone of the neural tube representing post-mitotic neurons. A small number of Olig2+/Hb9+ cells, or Olig2+/lslet1+ cells represent progenitor cells that are making the transition towards Hb9/MNR2+ neurons as they migrate away from the medial VZ to the peripheral MZ.

compared to the control ( $\mathrm{p}<0.001$ ) (Figure $2 \mathrm{j}$ ), consistent with previous findings that wt non-Tg astrocyte CM results in a highly significant increase in differentiated MNs [15].

To determine whether the increased proportion of differentiated MNs might be due, in part, to enhanced numbers of progenitors, we analysed expression of the b-HLH (basic helix-loop helix) transcription factor, Olig2 [20,25]. Many Olig2+ cells were detected within the cultures (Figure 2a,e). High magnification views after combinatorial labelling with Olig2, Hb9:eGFP and $\beta 3$-tubulin, or Olig2, Hb9:eGFP and Islet1 revealed that the vast majority of
Olig2+ cells did not co-express either $\beta 3$-tubulin (Figure 2c,d, red arrows) or Islet 1 (Figure 2h,i, red arrows). Quantitative analyses revealed that only a very minor proportion of Olig2 + cells co-expressed $\beta 3$-tubulin $(0.2 \%)$ or Islet $1(0.17 \%)$ (green arrow, Figure $2 \mathrm{e}-\mathrm{i})$. A significantly higher percentage of Olig2 $+\mathrm{MN}$-progenitor cells was detected in cultures grown in wt non-Tg astrocyte CM, compared to those in control medium (Figure 2k). Quantitative analyses showed that the percentage of Olig2+ MN-progenitors in wt non-Tg astrocyte CM was increased by 1.5 -fold compared to the 


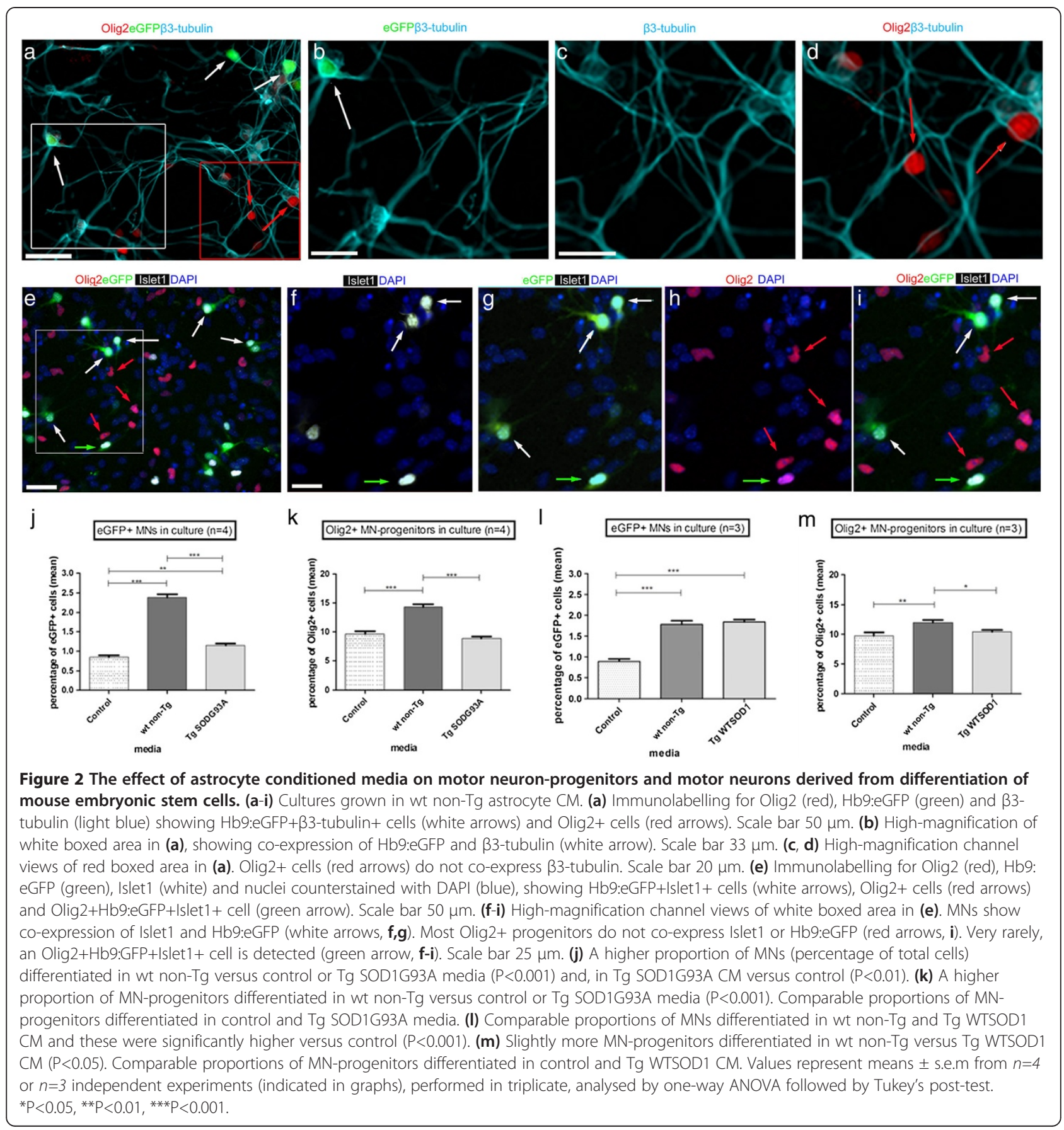

control $(\mathrm{p}<0.001)$. These data suggest that a primary effect of wt non-Tg astrocyte $\mathrm{CM}$ is to increase the number of MN progenitor cells. Importantly, moreover, culture with wt non- $\mathrm{Tg}$ astrocyte $\mathrm{CM}$ appeared to result in a more robust conversion of Olig2+ progenitors to MNs or survival of MNs. In the case of controls, approximately $8.8 \%$ of Olig2+ progenitors differentiated to MNs, whereas with wt non-Tg astrocyte CM, approximately $16.8 \%$ of Olig2+ progenitors differentiated to MNs. Thus, wt non-Tg astrocyte CM may promote the generation of differentiated
MNs in two ways: first, by increasing the numbers of progenitors, and second, by supporting their robust differentiation/survival.

In contrast to the effects of wt non- $\mathrm{Tg}$ astrocyte $\mathrm{CM}$, medium conditioned by astrocytes derived from transgenic mice expressing mutant SOD1-G93A (Tg SOD1-G93A) did not show a supportive effect on Olig2+ MN progenitors. Quantitative analyses showed that in Tg SOD1-G93A CM, the number of Olig2+ MN-progenitors was similar to those in the control ( $\mathrm{p}>0.05$, not significant), but decreased 
significantly $(\mathrm{p}<0.001$ : a 1.6 -fold decrease) compared to the littermate wt non-Tg CM (Figure 2k). Tg SOD1-G93A astrocyte $\mathrm{CM}$ similarly appeared far less able to support eGFP+ MNs, compared to wt non-Tg astrocyte CM. Quantitative analyses revealed a two-fold difference in the percentage of eGFP+ MNs in Tg SOD1-G93A CM compared to the littermate wt non-Tg CM (p <0.001) (Figure 2j). Notably, however, the efficiency of differentiation to MNs from Olig2+ progenitors in Tg SOD1-G93A CM appears comparable to that in wt non-Tg astrocyte CM (differentiation/ survival coefficient of $13 \%$ which is closer to the wt non-Tg astrocyte CM than to the control: see Methods). To determine whether Tg SOD1-G93A CM decreases the numbers of MN progenitors and MNs by increasing apoptosis, indicative of the presence of a toxic factor [15], we analysed chromatin condensation and nuclear fragmentation after DAPI-labelling [26]. Quantitative analyses revealed no statistically significant association of the number of apoptotic cells with any of the three media (Additional file 1: Figure S1). Taken together, these results indicate that, while CM from wt non- $\mathrm{Tg}$ astrocytes is strongly supportive of both Olig2+ MN progenitor cells and eGFP+ MNs, CM from Tg SOD1-G93A astrocytes appears to lack, in particular, a trophic support factor to motor neuron progenitors.

In order to confirm that the significant differences found in the proportion of Olig2+ MN-progenitor cells and eGFP+ MNs between the wt non-Tg astrocyte CM and the SOD1-G93A or the control medium were indeed due to the presence of the $G 93 A$ mutation and not to the presence of the transgene, a similar study was performed, this time using conditioned medium from transgenic astrocytes over-expressing human wild-type SOD1 ( $\mathrm{Tg}$ WTSOD1), or, for comparison, CM from their wt non- $\mathrm{Tg}$ littermates. As expected, the percentage of eGFP+ MNs detected after culture with either wt non-Tg or WTSOD1 $\mathrm{CM}$ was similar ( $\mathrm{p}>0.05$, not significant; Figure $2 \mathrm{l}$ ), while both media evoked a higher proportion of differentiated MNs than control medium (Figure 2l; $\mathrm{p}<0.001$; a twofold increase of eGFP+ MNs compared to the control; coefficient of differentiation is $11 \%$ for control; $16 \%$ for wt non-Tg; $18 \%$ for Tg WTSOD1). Similarly, there was a significant difference in the percentage of Olig2+ MNprogenitor cells in cultures grown in wt non- $\mathrm{Tg}$ astrocyte $\mathrm{CM}$ compared to control medium ( $\mathrm{p}<0.01$; Figure $2 \mathrm{~m}$ ). However, there was almost no difference in the proportion of Olig2+ cells supported through wt non-Tg murine astrocyte CM, compared to CM from Tg WTSOD1 littermates (Figure $2 \mathrm{~m} ; \mathrm{p}<0.05$ ). The similar proportion of Olig2+ progenitors and eGFP+ MNs generated in the presence of wt non-Tg and Tg WTSOD1 astrocyte CM (Figure 2l,m) suggests that the presence and overexpression of the human transgene in mouse astrocytes is not the reason for any observed differences in the numbers of eGFP+ MNs. Therefore, the difference in the proportion of eGFP+ MNs in the wt non-Tg and $\mathrm{Tg}$ SOD1-G93A astrocyte CM is likely to be due to the effect of the SOD1-G93A mutation.

\section{Neither wild type astrocyte CM nor mutant SOD1G93A astrocyte $\mathrm{CM}$ affects the generation of Lhx1/5+ interneurons from mouse ES cells}

We next examined whether the observed effects of $\mathrm{Tg}$ SOD1-G93A and wt non-Tg astrocyte CM were specific to $\mathrm{MN}$-progenitors and $\mathrm{MNs}$ or whether similar effects might be observed with other types of neurons. Analysis of Lhx $1 / 5+/ \mathrm{Hb} 9$ - interneurons (Lhx $1 / 5$ is the mammalian orthologue of chick Lim1/2, recognised by the anti-Lim1/ 2 antibody) $[27,28]$ revealed that the proportion of interneurons that differentiated in cultures was very similar for all three types of media examined. Thus, Tg SOD1-G93A $\mathrm{CM}$, littermate wt non- $\mathrm{Tg}$ astrocyte $\mathrm{CM}$ and control medium appeared to be equally supportive to interneurons ( $\mathrm{p}>0.05$; Figure $3 \mathrm{a}, \mathrm{b}$ ). This comparative study suggests that the supportive factors in wt non- $\mathrm{Tg}$ and $\mathrm{Tg}$ WTSOD1 astrocyte CM that are lacking in SOD1-G93A astrocyte $\mathrm{CM}$ may act only on MN progenitor cells and therefore particularly affect differentiated MNs.

\section{Discussion}

Published studies over the last few years have demonstrated negative effects of mutant SOD1 astrocytes on MN survival $[12-14,17]$ but no study has so far examined any potential effects on MN progenitor cells. The question of how astrocyte CM may impact on MN progenitors is important, both from the perspective of ex vivo differentiation strategies and, from the perspective of adult neurogenesis, since buffering of the niche from localised astrocytes may affect survival and differentiation efficiency of the progenitors. Here we demonstrate an impact of astrocyte $\mathrm{CM}$ on both $\mathrm{MN}$ progenitors and $\mathrm{MNs}$, and demonstrate that mutant SOD1 astrocytes fail, particularly, to provide survival/ trophic support to $\mathrm{MN}$ progenitors.

Our study reveals markedly higher numbers of Olig2+ $\mathrm{MN}$ progenitor cells and eGFP+ MNs in wt non-Tg astrocyte $\mathrm{CM}$ and consistently lower numbers in littermate $\mathrm{Tg}$ SOD1-G93A CM. In contrast, Tg WTSOD1 and littermate wt non-Tg CM have similar effects on eGFP+ MNs and Olig2+ MN-progenitors, indicating that our findings are likely due to the presence of mutant SOD1-G93A, rather than over-expressed SOD1 per se.

The significantly higher number of Olig2+ MN progenitor cells in the wt non- $\mathrm{Tg}$ astrocyte CM supports the idea that normal astrocytes favour MN development potentially by secreting supportive and/or trophic factors that promote $\mathrm{MN}$ progenitors. The positive role of astrocytes in promoting $\mathrm{MN}$ growth and maturation in in vitro cultures is already established [29]. Wild-type and mutant SOD1 astrocytes produce substantial amounts of functionally- 


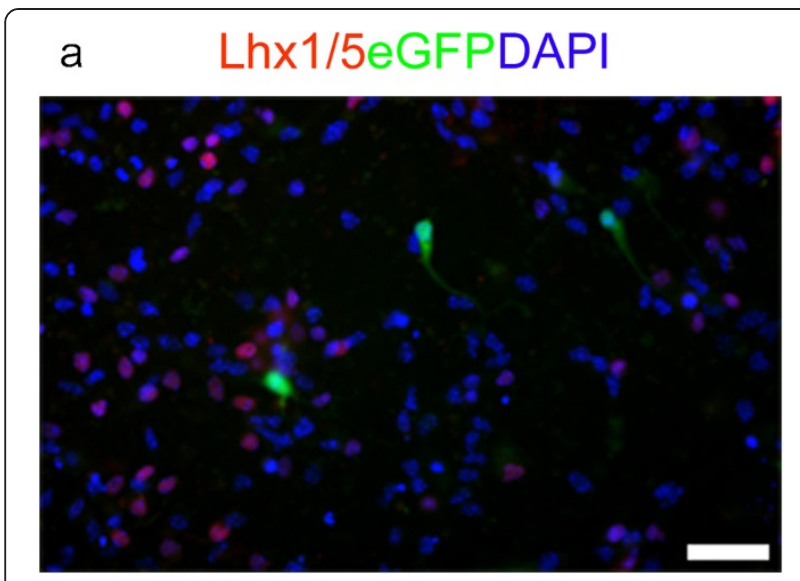

b

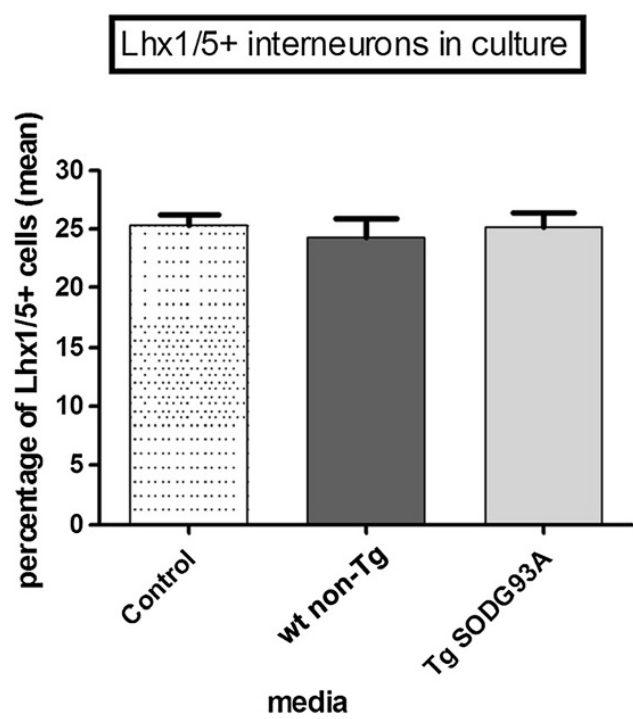

Figure 3 Examination of the effect of astrocyte conditioned media on interneurons derived from differentiation of mouse embryonic stem cells. (a) Double-labelled fluorescence image of culture grown in wt non-Tg astrocyte CM labelled for Lhx1/5 interneurons (red), Hb9:eGFP MNs (green) and DAPI for nuclei (blue). Scale bar $50 \mu \mathrm{m}$. (b) Analysis of the number of interneurons in control medium, wt non-Tg and Tg SOD1G93A CM. Data gathered from 2 independent experiments, each condition examined in triplicate and analysed by one-way ANOVA followed by Tukey's post-test. No significant differences in the proportion of interneurons were found between the three media.

variable factors that can positively or negatively affect MN survival. Such factors include trophic factors (GDNF -glial-derived neurotrophic factor, NGF) [30,31], pro-inflammatory/inflammatory proteins (iNOS -inducible nitric oxide synthase, cystatin- $C$, interferon- $\alpha$, prostaglandinD2) $[32,33]$, proteases (complement $3 \alpha, \beta$ ) and protease inhibitors, carrier proteins (apolipoprotein E, IBP-2 insulin-like growth factor binding protein-2), extracellular matrix binding proteins (cyclophilin-A) and anti-oxidant proteins (peroxiredoxins) [34]. Our study extends current knowledge by showing that astrocytes also positively affect
MN progenitor cells, potentially governing Olig2+ cellproliferation or Olig2+ cell-survival.

Unlike wt non-Tg astrocyte CM, Tg SOD1-G93A astrocyte CM does not favour MN progenitor cell growth: similar numbers of Olig2+ cells are found in Tg SOD1-G93A $\mathrm{CM}$ and in control non-conditioned medium. The relatively high numbers of differentiated MNs found with SOD1G93A CM, compared to control non-conditioned medium, however, suggests that the mutant astrocyte CM does support $\mathrm{MN}$ differentiation/survival. It is therefore likely that a number of the aforementioned factors that are produced by astrocytes and that affect $\mathrm{MN}$ survival are secreted in the conditioned medium and are also able to affect $\mathrm{MN}$ progenitor cell survival, growth or differentiation.

Our findings support a previous study that shows a halving in the number of primary and ES cell-derived MNs when cultured in Tg SOD1-G93A astrocyte CM compared to wt non-Tg astrocyte CM [15]. Our results differ from this study, however, in that they provide no evidence for $\mathrm{MN}$ death through increased apoptosis of MN progenitors or MNs: nuclear fragmentation was not increased by SOD1-G93A CM, nor do we observe a significant decrease in Olig2+ MN progenitor cells or MNs in Tg SOD1-G93A astrocyte CM compared to the baseline level in the presence of control non-conditioned medium. Why might our findings differ from this study, that demonstrated neurotoxicity by $S O D 1-G 93 A$ astrocytes mediated through the recruitment of the Bax-dependent death machinery? Notably, in our study, we analysed the effects of SOD1-G93A $\mathrm{CM}$ at an earlier time-point. Potentially, then, the neurotoxic effects of SOD1-G93A CM are realised later than the effects we examine here. While we cannot exclude the possibility that cell-death (through non-apoptotic pathways) contributes to the reduced numbers of MN progenitor cells, our data indicate that the mutant SOD1-G93A in astrocytes affects a secretory mechanism/product which renders the $\mathrm{CM}$ less supportive/trophic to $\mathrm{MN}$-progenitors, potentially affecting either Olig2+ cell-proliferation or Olig2+ cellsurvival. Thus, the beneficial effect observed with wt non-Tg $\mathrm{CM}$ is likely due to the presence of a supportive factor(s) that is absent or reduced from the Tg SOD1-G93A CM.

We also determined whether the effects of $\mathrm{Tg} S O D 1$ G93A and wt non-Tg astrocyte CM were specific to MNs and their progenitor cells by examining the effect on Lhx1/ 5+/Hb9- interneurons. Both the $\operatorname{Tg}$ SOD1-G93A and wt non- $\mathrm{Tg} C M$ were as trophic/supportive to interneurons as the control medium indicating that mutant SOD1-G93A in astrocytes did not affect interneurons and that the effects of wt non-Tg and Tg SOD1-G93A astrocyte CM were selective for MNs and MN progenitor cells. This is in agreement with Nagai et al. who reported no effects on primary spinal GABAergic or dorsal root ganglion neurons and ES cellderived interneurons grown in SOD1-G93A compared to non-Tg astrocyte CM [15]. 


\section{Conclusions}

Astrocytes are actively implicated in the neurodegeneration in ALS. The exact mechanisms by which mutant SOD1 in astrocytes can exert negative effects on $\mathrm{MNs}$ and $\mathrm{MN}$ progenitors still remain obscure, although existing evidence indicates that astrocytes exert their effects on MNs through several different mechanisms. Our study extends current knowledge by showing a negative effect of the SOD1-G93A mutation on Olig2+ MN-progenitor cells, possibly revealing new mechanisms of $\mathrm{MN}$ loss at the early stages of $\mathrm{MN}$ development.

\section{Methods \\ Antibodies}

Primary antibodies used were: anti-Olig2 rabbit polyclonal (Chemicon), anti-Lim1/2 (4F2) mouse monoclonal (Developmental Studies Hybridoma Bank), anti-Islet1 (4D5) mouse monoclonal (Developmental Studies Hybridoma Bank), anti-Hb9/MNR2 (5C10) mouse monoclonal (Developmental Studies Hybridoma Bank), $\beta 3$-tubulin (class 3 beta-tubulin) mouse monoclonal or rabbit polyclonal (Covance), anti-Ki67 rabbit polyclonal (Novocastra). Secondary antibodies used were anti-rabbit IgG-Alexa 594 and anti-mouse IgG-Alexa 594 (Molecular Probes), antimouse IgG-Cy5 (Jackson laboratories).

\section{Animal ethics statement}

All experiments were conducted according to the Animal (Scientific Procedures) Act 1986, under Project License 40/ 3089 reviewed and approved by the University of Sheffield Ethical Review Sub-Committee, and the UK Animal Procedures Committee (London, UK). The UK Home Office code of practice for the housing and care of animals used in scientific procedures was followed. We adhere to the ARRIVE guidelines for reporting animal research [35].

\section{Immunohistochemistry}

Mouse embryos at E10.5 were washed with PBS and fixed in $4 \%$ PFA for 2 hours at $4^{\circ} \mathrm{C}$, then rinsed with PBS and incubated in $0.2 \mathrm{M}$ phosphate buffer with $30 \%$ sucrose at $4^{\circ} \mathrm{C}$ overnight. Whole mount embryos were sectioned in slices of $15 \mu \mathrm{m}$ thickness using a cryostat at $-25^{\circ} \mathrm{C}$. Slices were collected on glass slides, allowed to dry for $30 \mathrm{~min}-1$ hour, then washed with PBS, permeabilised with $\mathrm{PBS} / 1 \%$ goat serum $/ 0.1 \%$ Triton $\mathrm{X}-100$ and incubated with primary antibodies at $4^{\circ} \mathrm{C}$ overnight. Incubation with secondary antibodies was performed at room temperature for 1 hour. The specimens were mounted in Vectashield Mounting medium with DAPI and sealed with cover glass. Microscopy was performed using ZEISS Axio Imager.Z1 microscope and AxioVision 4.6.3 software.

\section{Immunocytochemistry}

Cell cultures were fixed with $4 \%$ paraformaldehyde, permeabilised with PBS/ $2 \%$ goat serum/ $0.2 \%$ Triton X100 and incubated with primary antibodies at $4{ }^{\circ} \mathrm{C}$ overnight. Incubation with secondary antibodies was performed at room temperature for 1 hour and the specimens were mounted in Vectashield Mounting medium with DAPI. A ZEISS Axio Imager.Z1 microscope was used and images were captured using AxioVision 4.6.3 software. Islet1+, $\beta 3$ tubulin+ and Olig2+ cells were counted in 44 random fields and co-expression of the markers was estimated as a percentage of Olig2+ cells. For apoptosis assessment of Olig2+ and Hb9:eGFP+ cells, apoptotic nuclei exhibiting chromatin condensation and nuclear fragmentation after DAPI-labelling were counted in a total of 30 random fields per condition. For the conditioned media experiments, Olig2+, Hb9:GFP+ and Lhx1/5+ cells (the mammalian orthologue of chick Lim1/2, recognised by the anti-Lim1/2 antibody) were counted in 30 random fields per technical replica of each of the conditions (control, wt non-Tg or $\mathrm{Tg}$ ) and per experiment and calculated as the percentage of total cells (DAPI nuclei) per field. The percentages calculated from similar conditions within each experiment were pooled together. The mean percentages of positive cells for each marker and per condition were statistically analysed.

\section{Statistical analysis}

The data used for the statistical analyses were collected from $\mathrm{n}$ independent biological replicates as noted in the figure legends. In each biological replica, there were 3 technical replicas per condition (control medium, transgenic or wild-type non-transgenic mouse). Ten random fields per technical replica were used for cell counting. Thus at least 30 random fields were used per condition and per experiment; therefore at least 90 random fields were counted in total. Each field contains between 250300 cells. Therefore for each experiment, a sample size of at least 7500 cells was used per condition. Statistical analysis was performed using GraphPad Prism 5.0 software. Data were examined and fitted a normal distribution and therefore were analysed using one-way ANOVA followed by Tukey's multiple comparison post-test, with Confidence Interval $95 \%$ and alpha $=0.05$. Differences were considered significant for $\mathrm{P}$ values less than 0.05 . For apoptosis assessment, association of the number of apoptotic cells with the media was examined using the Chi-square test with 95\% Confidence Interval.

\section{Calculation of differentiation/survival coefficient}

For each condition (control, wt non- $\mathrm{Tg}$ or $\mathrm{Tg}$ conditioned medium) the number of Olig2+ and eGFP+ cells was used in the following formula:

Differentiation/survival coefficient $=\mathrm{Y} / \mathrm{X}$ *100; where $\mathrm{X}$ is the mean percentage of Olig2+ cells of the treatment 
group; $\mathrm{Y}$ is the mean percentage of eGFP+ cells of the treatment group.

The formula translates as follows: In a total of 100 cells, $\mathrm{X}$ cells are Olig2+ and Y cells differentiate to eGFP+ cells. Therefore, in 100 Olig2+ cells the proportion of cells to differentiate/survive towards eGFP+ cells is $\mathrm{Y} / \mathrm{X}{ }^{*} 100$.

\section{Preparation of SHH-N protein}

SHH-N protein containing culture supernatant was prepared as described previously [36]. Briefly, 3 days post transfection of a ShhN-pIRES2-eGFP construct into HEK/ $293 \mathrm{~T}$ cells, the culture supernatant was collected and concentrated up to $10 \times$ or $25 \times$ by repetitive centrifugation in columns of a $10 \mathrm{kDa}$ threshold (Amicon Bioseparations) according to the manufacturer's instructions.

\section{Preparation of murine primary astrocyte cultures}

Primary astrocyte cultures were prepared from the cortices of SOD1-G93A (B6SJLTg(SOD1*G93A)1Gur/J purchased from Jackson Laboratories), WT SOD1 and non-Tg littermate neonate 1-2 day old (p1-p2) mice. The total number of mice used were, eight Tg SOD1-G93A and ten wt non-Tg mice from four litters, six Tg WTSOD1 and six wt non-Tg from 3 litters. The cortices were stripped of meninges, washed and triturated in Hank's balanced salt solution (HBSS) with $\mathrm{Ca} 2+/ \mathrm{Mg} 2+$ containing $0.04 \%$ tryp$\sin$ (Sigma), $0.1 \mathrm{mg} / \mathrm{ml}$ collagenase (Calbiochem) and 0.05 $\mathrm{mg} / \mathrm{ml}$ DNaseI (Sigma). Following trituration, the enzymatic process was terminated by addition of an equal volume of complete medium [Dulbecco's modified Eagle's medium (DMEM, Cambrex), 10\% heat inactivated fetal calf serum (FCS, BioSera), 100 units $/ \mathrm{ml}$ penicillin and 100 $\mu \mathrm{g} / \mathrm{ml}$ streptomycin (Gibco)] and the cells plated in complete medium [10\% FCS, 2 mM L-glutamine, $100 \mathrm{U} / \mathrm{ml}$ Penicillin and $100 \mu \mathrm{g} / \mathrm{ml}$ Streptomycin] in a T25 (P1). The cell preparations were allowed to attach. After 48 hours, the medium with any cell debris was removed, cells were washed once with PBS and fresh complete medium was added. Astrocytes and microglia were allowed to grow to confluency for 2 weeks.

\section{Purification of astrocytes from microglia}

The overlying microglia cells were removed by shaking overnight on a rotatory shaker (Weiss Gallenkamp) at $37^{\circ} \mathrm{C}$ between 180-220 rpm. Pure astrocyte cultures were next established by mild trypsinisation $(0.25 \%$ trypsin and $1 \mathrm{mM}$ EDTA in HBSS $(-\mathrm{Ca} / \mathrm{Mg})$, added in a ratio of $1: 2$ with serum-free DMEM/F12), followed by trypsinisation with $2.5 \%$ trypsin in PBS and trituration up to single cells. Trypsin was inactivated with complete medium and the suspension was centrifuged. The astrocytes were plated in complete medium in T25 flasks (P2) and allowed to grow to confluency for 10 days before harvesting for conditioned medium.

\section{Preparation of astrocyte conditioned medium}

Conditioned medium was prepared by incubation with the astrocytes. The maintenance medium was removed from the cultures and astrocytes were rinsed with PBS. N2B27 medium (see below) was added and the medium was conditioned for 48 hours. Conditioned medium was collected, centrifuged to remove any cells present and stored at $4^{\circ} \mathrm{C}$ to be used immediately.

\section{Differentiation of mouse ES cells in monolayer culture}

MNs expressing eGFP were derived from the transgenic Hb9:eGFP mouse ES cell-line HBG3 [37] (generous gift from Professor Thomas Jessell, Columbia University). Mouse ES cells were removed from the flask by trypsinisation, resuspended in mESC-medium [DMEM $(4.5 \mathrm{~g} / \mathrm{L}$ glucose and $0.11 \mathrm{~g} / \mathrm{L}$ sodium pyruvate), Lglutamine $(2 \mathrm{mM})$, penicillin/streptomycin $(100 \mu \mathrm{g} / \mathrm{ml})$ (all from Gibco), 15\% FCS (Globepharm) and $0.1 \mathrm{mM}$ $\beta$-mercaptoethanol (Sigma)] and plated on tissue culture plastic substrate pre-coated with $0.1 \% \mathrm{w} / \mathrm{v}$ gelatin overnight. Mouse ES cells-medium was next replaced by N2B27 (1:1) medium. N2 consisted of DMEM:F12, BSA fraction- $\mathrm{V}(50 \mu \mathrm{g} / \mathrm{ml}$, Gibco), insulin $(25 \mu \mathrm{g} / \mathrm{ml})$, transferrin $(100 \mu \mathrm{g} / \mathrm{ml})$, progesterone $(20 \mathrm{nM})$, putrescine $(100 \mu \mathrm{M})$, sodium selenite $(20 \mathrm{nM})$ (all from Sigma), L-glutamine (2 mM), penicillin/streptomycin $(100 \mu \mathrm{g} / \mathrm{ml})$. B27 consisted of neurobasal medium (Gibco), B27 (1:50), L-glutamine $(2 \mathrm{mM})$, penicillin/streptomycin $(100 \mu \mathrm{g} / \mathrm{ml})$. The differentiating cells were cultured in N2B27 for 7 days, RA $(0.1 \mu \mathrm{M})$ and SHH-N (1x) added on days 3 and 4. On day 7 , the cells were trypsinised and replated on PDL $(10 \mu \mathrm{g} / \mathrm{ml}) /$ laminin $(5 \mu \mathrm{g} / \mathrm{ml})$ coated 8-well chamber slides at $5 \times 10^{4}$ cells $/ \mathrm{cm}^{2}$ in control N2B27 or astrocyte conditioned N2B27 supplemented with bFGF $(10 \mathrm{ng} / \mathrm{ml})$ for 2 days. After 2 days, bFGF was withdrawn and fresh control or conditioned N2B27 were added. The differentiation cultures were grown for another 3 days, prior to immunocytochemical analysis.

\section{Additional file}

Additional file 1: Figure S1. Examination of the effect of astrocyte conditioned media on the apoptosis of motor neuron-progenitor cells and motor neurons. (a, b) The apoptotic nuclei of Olig2+ MN-progenitor cells (a) and $\mathrm{Hb}$ 9:eGFP+ MNs (b) were counted in three independent experiments in a total of 30 random fields per condition. Statistically significant association of the number of apoptotic cells with the media was examined using Chi-square test with 95\% Confidence Interval (alpha=0.05). There is no statistically significant association between the level of apoptosis of Olig2+ cells ( $P$ value 0.2787) or eGFP+ MNs (P value 0.3028 ) with any of the three media. Apoptotic Olig2+ cells are 3.98\%, 3.22\% and $4.51 \%$ of the total Olig2+ cells in Tg SOD1G93A CM, wt non-Tg CM and control medium respectively. Apoptotic eGFP+ MNs are $6.32 \%, 3.11 \%$ and $3.82 \%$ of the total eGFP+ MNs in Tg SOD1G93A CM, wt non-Tg CM and control medium respectively.

\section{Abbreviations}

ALS: Amyotrophic lateral sclerosis; bHLH: basic Helix-Loop-Helix; CM: Conditioned medium; ES cells: Embryonic stem cells; MN: Motor neuron; 
NGF: Nerve growth factor; non-Tg: non-transgenic; SOD1: Superoxide dismutase-1; Tg: Transgenic; SVZ: Sub-ventricular zone; VZ: Ventricular zone; Wt: Wild-type.

\section{Competing interests}

The authors declare that they have no competing interests.

\section{Authors' contributions}

YAC designed and performed the experiments, performed the statistical analyses, interpreted the results and contributed to the manuscript preparation. KO and MP contributed to the design of the experiments, interpretation of results and manuscript preparation. PJS was the grant holder. PNM, MP and PJS supervised the study and contributed to the manuscript preparation. All authors read and approved the final manuscript.

\section{Acknowledgements}

We thank Professor Thomas Jessell (Columbia University) for the HBG3 mESC line. This study was supported by the Department of Neuroscience (PJ Shaw) and the MRC Centre for Developmental and Biomedical Genetics (M Placzek) of the University of Sheffield. PJS is supported as an NIHR Senior Investigator.

\section{Author details}

'Department of Neuroscience, Sheffield Institute for Translational Neuroscience (SITraN), University of Sheffield, Sheffield, S10 2HQ, UK. ${ }^{2}$ Department of Infection and Immunity, Faculty of Medicine, Dentistry and Health, University of Sheffield, Sheffield, S10 2HQ, UK. ${ }^{3} \mathrm{MRC}$ Centre for Developmental and Biomedical Genetics and Department of Biomedical Science, University of Sheffield, Sheffield, S10 2RX, UK.

Received: 11 June 2013 Accepted: 9 October 2013

Published: 17 October 2013

\section{References}

1. Hadano S, Hand CK, Osuga H, Yanagisawa Y, Otomo A, Devon RS, Miyamoto N, Showguchi-Miyata J, Okada Y, Singaraja R, et al: A gene encoding a putative GTPase regulator is mutated in familial amyotrophic lateral sclerosis 2. Nat Genet 2001, 29(2):166-173.

2. Kanekura K, Hashimoto Y, Niikura T, Aiso S, Matsuoka M, Nishimoto I: Alsin, the product of ALS2 gene, suppresses SOD1 mutant neurotoxicity through RhoGEF domain by interacting with SOD1 mutants. J Biol Chem 2004, 279(18):19247-19256.

3. Corrado L, Del Bo R, Castellotti B, Ratti A, Cereda C, Penco S, Soraru G, Carlomagno $Y$, Ghezzi S, Pensato V, et al: Mutations of FUS gene in sporadic amyotrophic lateral sclerosis. J Med Genet 2010, 47(3):190-194.

4. Kabashi E, Valdmanis PN, Dion P, Spiegelman D, McConkey BJ, Vande Velde C, Bouchard JP, Lacomblez L, Pochigaeva K, Salachas F, et al: TARDBP mutations in individuals with sporadic and familial amyotrophic lateral sclerosis. Nat Genet 2008, 40(5):572-574.

5. Corrado L, Ratti A, Gellera C, Buratti E, Castellotti B, Carlomagno Y, Ticozzi N, Mazzini L, Testa L, Taroni F, et al: High frequency of TARDBP gene mutations in Italian patients with amyotrophic lateral sclerosis. Hum Mutat 2009, 30(4):688-694.

6. Elden AC, Kim HJ, Hart MP, Chen-Plotkin AS, Johnson BS, Fang X, Armakola M, Geser F, Greene R, Lu MM, et al: Ataxin-2 intermediate-length polyglutamine expansions are associated with increased risk for ALS. Nature 2010, 466(7310):1069-1075.

7. Fernandez-Santiago R, Hoenig S, Lichtner P, Sperfeld AD, Sharma M, Berg D, Weichenrieder O, Illig T, Eger K, Meyer T, et al: Identification of novel Angiogenin (ANG) gene missense variants in German patients with amyotrophic lateral sclerosis. J Neurol 2009, 256(8):1337-1342.

8. DeJesus-Hernandez M, Mackenzie IR, Boeve BF, Boxer AL, Baker M, Rutherford NJ, Nicholson AM, Finch NA, Flynn H, Adamson J, et al: Expanded GGGGCC Hexanucleotide Repeat in Noncoding Region of C9ORF72 Causes Chromosome 9p-Linked FTD and ALS. Neuron 2011, 72(2):245-256.

9. Renton AE, Majounie E, Waite A, Simón-Sánchez J, Rollinson S, Raphae Gibbs J, Schymick JC, Laaksovirta H, Van Swieten JC, Myllykangas L, et al: A hexanucleotide repeat expansion in C9ORF72 is the cause of chromosome 9p21-linked ALS-FTD. Neuron 2011, 72(2):257-268.

10. Clement AM, Nguyen M, Roberts E, Garcia M, Boillee S, Rule M, McMahon AP, Doucette W, Siwek D, Ferrante RJ, et al: Wild-type nonneuronal cells extend survival of SOD1 mutant motor neurons in ALS mice. Science 2003, 302(5642):113-117.

11. Rothstein JD, Van Kammen M, Levey Al, Martin LJ, Kuncl RW: Selective loss of glial glutamate transporter GLT-1 in amyotrophic lateral sclerosis. Ann Neurol 1995, 38:73-84.

12. Yamanaka K, Chun SJ, Boillee S, Fujimori-Tonou N, Yamashita H, Gutmann DH, Takahashi R, Misawa H, Cleveland DW: Astrocytes as determinants of disease progression in inherited amyotrophic lateral sclerosis. Nat Neurosci 2008, 11:251-253.

13. Pehar M, Cassina P, Vargas MR, Castellanos R, Viera L, Beckman JS, Estevez $A G$, Barbeito L: Astrocytic production of nerve growth factor in motor neuron apoptosis: implications for amyotrophic lateral sclerosis. J Neurochemistry 2004, 89:464-473.

14. Ferraiuolo L, Higginbottom A, Heath PR, Barber S, Greenald D, Kirby J, Shaw PJ: Dysregulation of astrocyte-motoneuron cross-talk in mutant superoxide dismutase 1-related amyotrophic lateral sclerosis. Brain 2011 134(Pt 9):2627-2641.

15. Nagai M, Re DB, Nagata T, Chalazonitis A, Jessell TM, Wichterle $H$, Przedborski S: Astrocytes expressing ALS-linked mutated SOD1 release factors selectively toxic to motor neurons. Nat Neurosci 2007, 10:615-622.

16. Di Giorgio FP, Carrasco MA, Siao MC, Maniatis T, Eggan K: Non-cell autonomous effect of glia on motor neurons in an embryonic stem-cell based ALS model. Nat Neurosci 2007, 10:608-614.

17. Lepore AC, Rauck B, Dejea C, Pardo AC, Rao MS, Rothstein JD, Maragakis NJ: Focal transplantation-based astrocyte replacement is neuroprotective in a model of motor neuron disease. Nat Neurosci 2008, 11:1294-1301.

18. Pytte C, Yu YL, Wildstein S, George S, Kirn JR: Adult neuron addition to the zebra finch song motor pathway correlates with the rate and extent of recovery from botox-induced paralysis of the vocal muscles. J Neurosci 2011, 31(47):16958-16968.

19. Reimer MM, Norris A, Ohnmacht J, Patani R, Zhong Z, Dias TB, Kuscha V, Scott AL, Chen Y-C, Rozov S, et al: Dopamine from the brain promotes spinal motor neuron generation during development and adult regeneration. Dev Cell 2013, 25(5):478-491.

20. Novitch BG, Wichterle $H$, Jessell TM, Sockanathan S: A requirement for retinoic acid-mediated transcriptional activation in ventral neural patterning and motor neuron specification. Neuron 2003, 40(1):81-95.

21. Lee S, Pfaff S: Synchronization of neurogenesis and motor neuron specification by direct coupling of bHLH and homeodomain transcription factors. Neuron 2003, 38(5):731-745.

22. Lee SK, Jurata LW, Funahashi J, Ruiz EC, Pfaff SL: Analysis of embryonic motoneuron gene regulation: derepression of general activators function in concert with enhancer factors. Development 2004 131(14):3295-3306

23. Novitch $\mathrm{BG}$, Chen Al, Jessell TM: Coordinate regulation of motor neuron subtype identity and pan-neuronal properties by the bHLH repressor Olig2. Neuron 2001, 31(5):773-789.

24. Gaber ZB, Novitch BG: All the embryo's a stage, and Olig2 in its time plays many parts. Neuron 2011, 69(5):833-835.

25. Lee SK, Lee B, Ruiz EC, Pfaff SL: Olig2 and Ngn2 function in opposition to modulate gene expression in motor neuron progenitor cells. Genes Dev 2005, 19(2):282-294.

26. Doonan F, Cotter TG: Morphological assessment of apoptosis. Methods 2008, 44(3):200-204.

27. Gross MK, Dottori M, Goulding M: Lbx1 specifies somatosensory association interneurons in the dorsal spinal cord. Neuron 2002, 34(4):535-549.

28. Muller T, Brohmann H, Pierani A, Heppenstall PA, Lewin GR, Jessell TM, Birchmeier $\mathrm{C}$ : The homeodomain factor Ibx1 distinguishes two major programs of neuronal differentiation in the dorsal spinal cord. Neuron 2002, 34(4):551-562.

29. Ullian EM, Harris BT, Wu A, Chan JR, Barres BA: Schwann cells and astrocytes induce synapse formation by spinal motor neurons in culture. Mol Cell Neurosci 2004, 25:241-251.

30. Suzuki M, McHugh J, Tork C, Shelley B, Klein SM, Aebischer P, Svendsen CN: GDNF secreting human neural progenitor cells protect dying motor neurons, but not their projection to muscle, in a rat model of familial ALS. PLOS ONE 2007, 2(8):e689.

31. Pehar M, Cassina P, Vargas MR, Castellanos R, Viera L, Beckman JS, Estevez $A G$, Barbeito L: Astrocytic production of nerve growth factor in motor neuron apoptosis: implications for amyotrophic lateral sclerosis. J Neurochem 2004, 89(2):464-473. 
32. Marchetto MC, Muotri AR, Mu Y, Smith AM, Cezar GG, Gage FH: Non-cellautonomous effect of human SOD1 G37R astrocytes on motor neurons derived from human embryonic stem cells. Cell Stem Cell 2008, 3(6):649-657.

33. Di Giorgio FP, Boulting GL, Bobrowicz S, Eggan KC: Human embryonic stem cell-derived motor neurons are sensitive to the toxic effect of glial cells carrying an ALS-causing mutation. Cell Stem Cell 2008, 3(6):637-648.

34. Lafon-Cazal M, Adjali O, Galeotti N, Poncet J, Jouin P, Homburger V, Bockaert J, Marin P: Proteomic analysis of astrocytic secretion in the mouse. Comparison with the cerebrospinal fluid proteome. J Biol Chem 2003, 278:24438-24448.

35. Kilkenny C, Browne WJ, Cuthill IC, Emerson M, Altman DG: Improving bioscience research reporting: the ARRIVE guidelines for reporting animal research. PLOS Biol 2010, 8(6):e1000412.

36. Ohyama K, Ellis P, Kimura S, Placzek M: Directed differentiation of neural cells to hypothalamic dopaminergic neurons. Development 2005, 132(23):5185-5197.

37. Wichterle H, Lieberam I, Porter JA, Jessell TM: Directed differentiation of embryonic stem cells into motor neurons. Cell 2002, 110(3):385-397.

doi:10.1186/1471-2202-14-126

Cite this article as: Christou et al: Wild-type but not mutant SOD1 transgenic astrocytes promote the efficient generation of motor neuron progenitors from mouse embryonic stem cells. BMC Neuroscience 2013 14:126

\section{Submit your next manuscript to BioMed Central and take full advantage of:}

- Convenient online submission

- Thorough peer review

- No space constraints or color figure charges

- Immediate publication on acceptance

- Inclusion in PubMed, CAS, Scopus and Google Scholar

- Research which is freely available for redistribution 